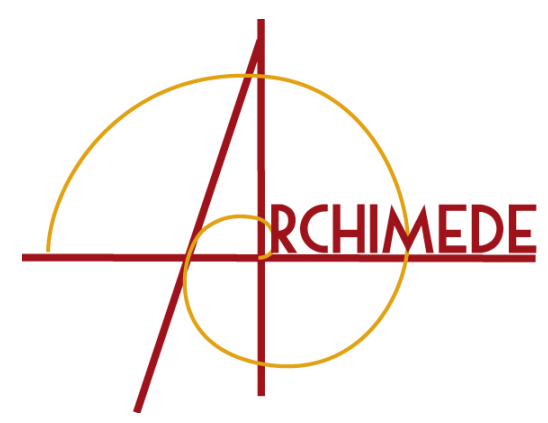

\title{
LE SACRIFICE DANS LE MONDE GREC ET SES INTERPRÉTATIONS
}

\author{
Anne JACQUEMIN \\ Université de Strasbourg \\ UMR 7044 - Archimède \\ jacquemi@unistra.fr
}

\section{RÉSUMÉ}

Le sacrifice sanglant est l'acte majeur du rituel grec. Si le récit fondateur principal remonte à Hésiode (sacrifice de Prométhée), d'autres récits, plus tardifs, ont développé le thème de la culpabilité liée à l'acte (Porphyre). La recherche s'était attachée dans les années 1970 au thème de la séparation dégagé du récit hésiodique, ainsi qu'à celui du consentement de la victime retrouvé dans les représentations de sacrifice (École de Paris : J.-P. Vernant, St. Georgoudi, J.-L. Durand). L'accent est plus mis aujourd'hui sur la participation des hommes (et des dieux) à l'acte rituel (R. Parker). Il convient donc de s'interroger sur ce qui fait qu'une même documentation peut donner lieu à des interprétations divergentes et le faire en examinant le rôle joué par les focales et la place

Mots-CLÉs

Animal, dieux, hommes, sacrifice, viande. tenue par le découpage opéré dans le champ des données, sans oublier la part qui peut être celle de l'inscription dans le temps des sources utilisées.
Sacrifice in the Greek world and its interpretations. Animal-sacrifice is the major ritual practice for the Greeks. The aitiological discourse is Hesiodic (sacrifice by Prometheus), but others later tales deal with the motive of guilt (Porphyrus). Research in the seventies aimed principally to the Hesiodic scheme of human separation from the gods and to the «yes » of the animal to its sacrifical death as it was found in figured representations (École de Paris : J.-P. Vernant, St. Georgoudi, J.-L. Durand). Today the theme of common participation to the ritual practice - mortals as well as gods - is more put in light ( $R$. Parker). So it's time to examine why the same documentation can lead to divergent interpretations, scrutinizing the role played by the focals, the selection in the data, but also by the historical moment where the used sources come in sight.
Keywords Animal, gods, human beings, sacrifice, meat. 
Les quelques réflexions qui suivent sont une invitation

à la prudence face aux interprétations hâtives, au désir de tout comprendre, de tout assimiler.

Le sacrifice sanglant (thusia) qui est le plus important des actes par lequel les Grecs entraient en relation avec les puissances surnaturelles, dieux, héros et daimones se prête particulièrement bien à l'exercice de mise en perspective critique [1].

Si sacrificium en latin qui a donné le français « sacrifice » désigne l'acte qui rend sacer, consacré à une divinité de façon positive ou négative, puisque sacer signifie également maudit, l'équivalent grec du verbe sacrificare, hiereuein, « consacrer », transformer un animal en hiereion, en animal consacré, est moins fréquent que le verbe thueien dont dérive le nom le plus courant signifiant le sacrifice en grec, thusia. Thuein qui est formé sur la racine indo-européenne signifiant «fumer, faire fumer » renvoie à la combustion qui réalise la consécration [2]. Ce qui explique que ce verbe puisse être employé pour des sacrifices non sanglants, comme des offrandes de gâteaux : il a pour complément soit ce qu'on offre (bœuf, chèvre, farine, miel...) soit la fête qu'on célèbre (Lukaia, Herakleia - la fête de Zeus du mont Lycée, la fête d'Héraclès). Quoiqu'il soit d'usage fréquent de traduire en français hiereion par « victime » et que ce dernier terme vienne bien du latin victima qui désigne l'animal de sacrifice [3], les connotations liées à la notion de « victime » font qu'il est préférable, comme l'ont bien montré P. Brulé et R. Touze [4], d'éviter ce mot.

Le sacrifice sanglant est une réalité présente dans les plus anciens textes grecs conservés que sont les épopées homériques : au chant I de l'Iliade [5], quand le prêtre Chrysès rappelle à Apollon Smintheus les gras cuisseaux de taureaux et de chèvres qu'il a brûlés pour lui, il est clair que la notion d'un partage des viandes entre les dieux et les hommes est présente à l'esprit du poète. Un peu plus

[1] Compte tenu de la dimension interdisciplinaire du dossier, les mots grecs sont translittérés et les textes traduits.

[2] Chantraine 2009, s.u. thuô.

[3] Le victimaire (victimarius) est celui qui vend des animaux de sacrifice ou celui qui prépare ce qui est nécessaire au sacrifice.

[4] BRULÉ, Touze 2008. Voir aussi la préface de R. Parker à BRULÉ, MEHL 2008 , p. III-V, qui rappelle la difficulté que rencontrent les historiens et archéologues pour ne pas employer ce mot de « victime » qui vient spontanément, même sous la plume de qui est le plus conscient de I'absence de notion de victimisation chez les Grecs.

[5] Iliade, I. 37-42 tard, lorsque Ulysse rend, au nom d'Agamemnon, Chryséis à son père, il offre pour les Achéens une hécatombe à Apollon par l'intermédiaire de Chrysès :

Après avoir prié et répandu les grains d'orge, ils redressèrent d'abord les têtes des victimes, les égorgèrent, les dépecèrent, découpèrent les cuisseaux (mèroi), les recouvrirent de graisse sur les deux faces et posèrent dessus la chair crue. Puis le vieillard les fit cuire sur des bûches et, sur elles, répandit du vin flamboyant. Les jeunes gens, auprès de lui, tenaient en main la fourchette à cinq dents. Une fois que les cuisseaux furent consumés et qu'ils eurent mangé les viscères (splankhna), ils découpèrent menu le reste, l'enfilèrent sur des broches, le firent rôtir à point et retirèrent le tout du feu. Ce travail terminé et le banquet (daïs) préparé, ils banquetèrent et les cœurs n'eurent point à se plaindre d'un repas où chacun avait eu sa part. Puis, quand on eut fait taire la soif et la faim, les jeunes gens remplirent les cratères et versèrent à tous dans leurs coupes le contenu d'une libation. Et, tout le jour, en chœur, pour apaiser le dieu, les fils des Achéens chantèrent un beau péan en I'honneur de Celui qui repousse au loin des fléaux (Hékaergos) [6].

S'il y a bien là un partage des viandes entre Apollon, son prêtre et les membres de la délégation achéenne, il convient de noter que, dans les poèmes homériques, la consommation de viande n'a pas toujours lieu dans un contexte sacrificiel. Si rien n'est dit de l'origine des dos de brebis et de chèvres et de I'échine de porc qu'Achille offre aux envoyés d'Agamemnon [7], en revanche, il est précisé qu'il fit tuer une brebis pour le repas de Priam [8]. L'Odyssée présente un authentique sacrifice de taureaux à Poséidon et un sacrifice à Athéna d'une génisse aux cornes plaquées d'or, tous deux offerts par Nestor [9].

[6] Iliade, I 447-474.

[7] Iliade, IX, 205-217 : Patrocle place sur un billot les pièces de viande qu'Achille et Automédon découpe afin de faire des broches qui sont cuites à la braise. Il s'agit d'une consommation purement profane où les dieux n'ont pas leur part.

[8] Iliade, XXIV, 621-627. Dans ce cas également, les dieux ne sont aucunement conviés à ce repas de viande en brochettes et de pain.

[9] Odyssée, III, 4-9 et 29-64 (sacrifice à Poséidon, partage des viandes entre le dieu et les hommes et festin) ; 419-463 (sacrifice de la génisse à Athéna, après que Nestor a découvert que la déesse a pris part sous l'apparence humaine de Mentor à la première consommation de viande). 
Le second fait l'objet d'une description détaillée où I'on retrouve les mêmes séquences que dans le sacrifice du chant I de I'Iliade. Un sacrifice d'un tout autre type est accompli par Ulysse quand il se rend chez les morts pour consulter le devin Tirésias [10] : les morts viennent boire le sang de l'agneau et de la brebis dont la chair est brûlée, tandis que sont invoqués Hadès et Perséphone. Il ne saurait être question d'une communion entre vivants et morts par le partage et la consommation d'une même viande. Cette idée est rendue de façon très sensible par un cratère attique à figures rouges, œuvre du milieu du ve siècle attribuée au Peintre de Lycaon et conservée au Musée des Beaux-Arts de Boston [11].

Le sacrifice sanglant suivi de consommation de viande que décrit Homère est le sacrifice grec courant, bien connu de I'auditoire de l'aède. Les modernes ont vu le récit originel (aition) de cet acte rituel [12] dans les vers d'Hésiode qui expliquent la séparation instaurée entre les dieux et les hommes à la suite du sacrifice accompli à Mékôné, dans la région de Sicyone, au nord-est du Péloponnèse, par cet intermédiaire entre les dieux et les hommes qu'est Prométhée.

C'était aux temps où se départageaient dieux et hommes mortels à Mékôné. En ce jour-là, Prométhée avait, d'un cœur empressé, partagé un bœuf énorme, qu'il avait ensuite placé devant tous. Il cherchait à tromper la pensée de Zeus : pour l'un des deux partis, il avait mis sous la peau chairs et entrailles lourdes de graisse, puis recouvert le tout de la panse du bœuf ; pour l'autre, il avait, par une ruse perfide, disposé en un tas les os blancs du bœuf, puis recouvert le tout de graisse blanche; Sur quoi, le père des dieux et des hommes lui dit : «O fils de Japet, noble sire entre tous, tu as, bel ami, été bien partial en faisant les lots ».

Ainsi, railleur, parlait Zeus aux conseils éternels. Et Prométhée aux pensers fourbes lui répondit avec un léger sourire, soucieux de sa ruse perfide : «Zeus très grand, le plus glorieux des dieux toujours vivants, choisis donc de ces deux parts celle que ton cœur t'indique en ta poitrine ».

[10] Odyssée, X, 514-538 (conseils de Circé) ; XI, 23-30 (Ulysse accomplit le sacrifice selon les instructions).

[11] William Amory Gardner Fund, 34-79. Reproduit dans CомSTOCK s.d., n48. www.mfa.org/collections/object/jar-pelike-with-odysseusand-elpenor-in-the-underworld-153840.

[12] Le poète béotien limite la portée de son récit au seul usage de brûler les os sur l'autel (Théogonie, 556-557).

[13] Théogonie, 535-560 (tr. P. Mazon, CUF, 1928).

[14] VeRnANT 1972.

[15] Le vol du feu et sa transmission aux hommes conduisit au châtiment de Prométhée (voir Eschyle, Prométhée enchaîné), auquel Zeus mit fin, en envoyant Héraclès délivrer le Titan et tuer l'aigle qui dévorait le foie de ce dernier, au terme d'un accord qui permettait au souverain des dieux de connaître le nom de celle dont le fils, plus puissant que son père, réintroduirait le chaos dans l'univers. Quant aux hommes, ils furent
Il dit, le cœur plein de fourbe, et Zeus aux conseils éternels comprit la ruse et sut la reconnaître. Mais déjà en son cœur, il méditait la ruine des mortels, tout comme en fait il devait l'achever. De ses deux mains, il souleva la graisse blanche, et la colère emplit son âme, tandis que la bile montait en son cœur à la vue des os blancs du bœuf, trahissant la ruse perfide - Et aussi bien est-ce pourquoi, sur la terre, les fils des hommes brûlent aux immortels les os blancs sur les autels odorants. - Et, indigné, I'assembleur des nuées, Zeus dit : «Ah! Fils de Japet, qui en sais plus que nul au monde. Je le vois, bel ami, tu n'as pas encore oublié la ruse perfide » [13].

Pour J.-P. Vernant [14], ce récit, qui est lié au mythe de Pandôra, la première femme, est non seulement l'étiologie du sacrifice sanglant, mais aussi « une analyse du statut définissant la condition humaine », à savoir une séparation radicale d'avec les Bienheureux qui étaient à l'origine les commensaux des humains. Zeus s'est en effet vengé du tour qu'avait pensé lui jouer Prométhée en retirant le feu aux hommes que le partage était censé avantager [15]. Désormais, ces derniers mangent la chair d'un animal que la vie a quitté ; mortels, ils se nourrissent de chair morte ; les dieux se repaissent, eux, de la fumée odorante des os imputrescibles.

Hésiode ne mentionne pas les splankhna qui font pourtant I'objet d'une consommation rituelle par les hommes, sans être de la viande (sarkes, kréa). Pour J.-P. Vernant, il ne peut s'agir d'un oubli et c'est volontairement que le poète passe sous silence ce qui rendrait la séparation moins tranchée entre les dieux et les hommes. Les splankhna sont en effet des organes sanguins (cœur, foie, rate, pancréas) qui sont rôtis sur la flamme de l'autel : quoiqu'ils soient consommés par les hommes, ils sont du côté du divin, comme le montre le lieu de leur cuisine, à la différence des gigots mous qui sont bouillis dans les chaudrons avant d'être embrochés pour être rôtis sur des foyers au sol, à l'extérieur de l'espace rituel proprement dit [16]. Hésiode joue de l'ambiguïté du terme enkata (viscères) qui désigne aussi bien ces organes sanguins que l'estomac et les intestins voués à la digestion (tripes).

punis par l'introduction dans le monde d'un beau mal, fléau des hommes (Hésiode, Théogonie, 561-612 - le poète fait de la vision par Zeus du feu de nouveau entre les mains des hommes la cause directe de la création de Pandôra). Le poète ne présente pas vraiment la première société humaine qui n'aurait été alors constituée que d'éléments masculins : il est vrai que la commensalité avec les dieux devait préserver ses membres de la mort et de la nécessité de la reproduction.

[16] Cette cuisson avait lieu dans les espaces périphériques des sanctuaires. Une étude en cours menée dans le cadre d'un programme de I'École française d'Athènes par S. Huber, A. Jacquemin et D. Laroche s'est donné pour but de retrouver les lieux des différents moments du sacrifice à Apollon Pythien. Dans certains cas, la consommation se fait d'ailleurs hors du sanctuaire, comme lors des Panathénées athéniennes où les parts de viande qui ne sont pas réservées à ceux qui ont participé à la procession sont distribuées au Céramique, au Pompeion (IG II 334 : voir BRULÉ 2007a). 
Le sacrifice sanglant serait ainsi un rappel de la condition de I'homme mangeur de blé, voué à la mort par sa consommation de chair morte. Puisqu'il n'y a plus de véritable commensalité entre dieux et hommes et que chacun mange une part différente, le sacrifice sépare plus qu'il unit. Les conclusions de J.-P. Vernant ont été partagées par ceux qui, autour de lui, se sont intéressés à la question, notamment $M$. Detienne, St. Georgoudi et J.-L. Durand. Ces deux derniers ont étudié principalement l'animal sacrifié et le consentement qu'il était censé donner à son immolation.

C'est dans cette perspective que J.-L. Durand [17] a traité le rituel athénien des Bouphonies, un sacrifice accompli sur I'acropole en I'honneur de Zeus Polieus, dont il a analysé le mythe fondateur tel qu'il est rapporté par Porphyre citant le traité de Théophraste intitulé Sur la piété [18].

Dans l'ancien temps, les hommes sacrifiaient aux dieux les produits de leurs récoltes, mais point d'animaux ; ils ne les utilisaient pas non plus pour leur nourriture personnelle. Au cours, dit-on, d'un sacrifice collectif qui avait lieu à Athènes, un certain Sôpatros qui n'était pas du pays, mais qui cultivait la terre à travers l'Attique, avait déposé sur la table, bien en évidence, un gâteau et les pâtes pour les sacrifier aux dieux, quand un des bœufs au retour du travail, en rentrant, dévora une partie des offrandes, et piétina le reste. Pris d'une violente colère devant ce qui se passait, I'homme, alors qu'une hache était aiguisée non loin de là, l'arracha et abattit le bœuf. Quand la bête fut morte, I'homme, revenant de sa colère, prit conscience de I'acte qu'il venait de commettre. Il donna une sépulture au bœuf, puis, partant de lui-même en exil, comme un homme qui se sent coupable d'impiété, il s'enfuit en Crète. Or une sécheresse vint à sévir avec une terrible pénurie de récoltes. À la délégation commune venue consulter le dieu, la Pythie répondit que l'exilé de Crète mettrait fin à tout cela et que, s'ils punissaient le meurtrier, s'ils remettaient sur pied le mort à l'intérieur du même sacrifice que celui où précisément il avait perdu la vie, les choses iraient mieux pour eux à condition de consommer le mort et de ne pas s'en faire scrupule. On se mit alors en quête de Sôpatros, le responsable de l'affaire. Sôpatros pensa qu'il serait délivré de la situation pénible où le mettait son état d'impureté si tout le monde faisait collectivement comme lui. Il déclara donc à ceux qui était venu le chercher qu'il fallait qu'un bœuf fût mis à bas par la cité. Comme ils étaient embarrassés pour choisir qui abattrait l'animal, il leur offrit de le faire si, après

[17] DURAND 1986

[18] Porphyre, Sur I'abstinence, II, 28, 4 - 31,1 (tr. ]. Bouffartigue et M. Patillon dans leur édition de Porphyre de la Collection des Universités de France, revue par J.-L. Durand dans DuRAND 1986, p. 45-46).

[19] Quant à l'ouvrage du péripatéticien Théophraste, son titre invite à y voir une recherche sur la vraie piété qui est celle du cœur et non celle qui se manifeste dans la somptuosité des sacrifices lui avoir conféré le droit de cité, ils mettaient le meurtre en commun. Ils acceptèrent, et, à leur retour dans la cité, ils organisèrent de la façon suivante la pratique telle qu'elle est encore en vigueur chez eux.

Ils choisirent des jeunes filles comme porteuses d'eau ; ces jeunes filles apportent l'eau pour aiguiser la hache et le couteau. Les instruments aiguisés, un second tendit la hache, un autre abattit le bœuf et un autre l'égorgea. Puis d'autres encore l'écorchèrent, et tout le monde en goûta. Après ces opérations, on cousit le peau du bœuf, on la bourra de foin et on la mit sur pied en lui donna l'allure même qu'avait l'animal quand il était vivant, puis on I'attela à une araire, comme si on le mettait au travail. Ensuite on procéda au jugement du meurtre et tous ceux qui avaient en commun pris part à l'opération furent cités pour se justifier. Ceux qui avaient aiguisé désignèrent celui qui avait tendu la hache ; celui-ci I'homme qui avait égorgé I'animal, celui qui avait accompli cette action, désigna le couteau ; ce dernier, n'ayant pas le pouvoir de parler, fut accusé de meurtre.

Il ne faut oublier en lisant ce texte qu'il a été conservé par un philosophe néo-platonicien, auteur d'une vie de Pythagore, qui était opposé à la consommation d'êtres vivants et qui ne pouvait donc qu'être un adversaire du sacrifice sanglant [19]. Dans ce récit, le thème de la faute tient une place importante : le bœuf est coupable de gourmandise [20] ; I'homme sacrificateur cherche à échapper à sa culpabilité en la transférant à la hache qui a tué ou à l'animal qui méritait un châtiment. Dans ce contexte, le consentement de I'animal à son sort libère I'homme. Il convient cependant à ce point de revenir au récit hésiodique : il n'y a aucun sentiment de culpabilité, parce qu'il n'y a pas place pour lui dans le récit qui n'évoque pas la mise à mort de l'animal, mais passe tout de suite à son partage par Prométhée.

Séparation nette entre les hommes et les dieux, malaise face au sacrifice sanglant et stratagèmes pour échapper à la mauvaise conscience sont les thèmes qui ont été largement développés dans les études menées autour de J.-P. Vernant, dans des travaux qui ont exploité les textes littéraires et la documentation iconographique. Le colloque organisé en 1981 à Vandœuvres-Genève par la fondation Hardt se plaçait sous le patronage de ce savant qui donna la contribution inaugurale intitulée «Théorie générale du sacrifice et de la mise à mort dans la thusia grecque »[21]. Il y développe la question du moment dramatique de la mise à mort, en montrant que la violence et le meurtre sont au cœur du

sanglants, quel que soit le rôle qu'ils jouent dans la religion de la cité.

[20] C'est aussi le cas d'autres animaux (chèvre, porc...) dans des récits de premiers sacrifices à Déméter ou à Dionysos que nous connaissons également par Porphyre : ils ont tous mangé ce qui ne devait pas être leur nourriture. Voir sur ce point DURAND 1986, p. 14-21.

[21] VERNANT 1981. 
sacrifice. Ce type d'analyse du sacrifice devint quasiment la doxa, du moins en France, comme on peut le voir dans les manuels d'histoire de la religion grecque [22].

Dans les mêmes années, des recherches menées, entre autres, par des savants suédois [23] se sont intéressées plus à la matérialité des sacrifices, en utilisant les règlements souvent encore appelés « lois sacrées », les calendriers sacrificiels et les comptes des sanctuaires qui fournissent des renseignements sur les types d'animaux sacrifiés (sexe, âge, couleur de la robe, prix minimum), les données archéologiques (autels et espaces sacrificiels), et, plus récemment les données ostéologiques. C'est dans cette perspective que se situait le colloque L'espace sacrificiel organisé à Lyon en 1988 [24].

Au XXI ${ }^{\mathrm{e}}$ siècle, on assiste à une sorte de synthèse des deux approches. Lorsque L. Bruit-Zaidman écrit « d'un côté la construction théologique d'Hésiode ..., de l'autre la réalité politique de la pratique de la cité ... » [25], elle essaie encore de garder unies les deux conceptions. Mais un certain nombre d'évidences sont remises en cause par ceux-là même qui les avaient défendues une vingtaine d'années auparavant. Stella Georgoudi revient sur la cuisine du sacrifice et reprend la question du consentement de l'animal sacrifié dans un ouvrage collectif [26] et dans les actes de la section consacrée au sacrifice antique de la Quatrième conférence celtique de Lampeter (Pays de Galles) en 2006 [27]. Il faut reconnaître que certains avaient des doutes depuis longtemps face à des glissements opérés, souvent inconsciemment, pour donner plus de force à la théorie : ainsi le geste, parfaitement attesté par les sources, de l'aspersion de l'animal, dans lequel R. Ginouvès voyait un simple acte de purification par l'eau [28], devenait pour W. Burkert [29] une preuve à l'appui de la théorie de l'acquiescement de l'animal à son sort, qui avait été déjà exprimée par K. Meuli [30] et par J. Rudhardt [31]. En 1959, dans son étude sur l'iconogra- phie des animaux de sacrifice, F. Van Straten [32] s'étonnait de l'absence d'intérêt des imagiers grecs pour une donnée aussi fondamentale. Cette remarque lui valut dans le compte rendu de son livre par $\mathrm{P}$. Bonnechère le rappel des « nombreuses allusions textuelles » à ce fameux acquiescement. C'est ce qui a conduit St. Georgoudi à regarder les textes en question pour découvrir qu'ils ne sont en fait que deux et qu'il s'agit dans les deux cas de scholies, l'une à la Paix d'Aristophane, I'autre aux Argonautiques d'Apollonios de Rhodes. De plus, ces textes se bornent à exposer la bonne volonté de I'animal, sa piété envers les dieux et ne font aucune allusion à la culpabilité dont l'homme serait ainsi déchargé. St. Georgoudi a alors posé la question de l'utilisation du témoignage de Porphyre pour conclure que ce penseur avait fait l'objet d'une « utilisation parfois désinvolte de [ses] récits et de [ses] positions philosophiques » qui traduisent en fait le point de vue des sectes pythagoriciennes et non le discours commun des Grecs [33]. Le même constat doit être fait en ce qui concerne le récit d'Élien à propos du sanctuaire d'Aphrodite à Éryx [34] où les animaux viennent d'offrir d'eux-mêmes [35].

Les études sur le sacrifice en Grèce se placent aujourd'hui dans une approche plus fidèle à ce que la documentation dans sa diversité donne à voir du sacrifice grec. Ainsi, dans la conférence inaugurale du colloque de l'Institut suédois en avril 2008 [36], R. Parker a mis l'accent sur l'aspect de communion du sacrifice grec selon deux axes, vertical entre les hommes et les dieux et horizontal entre les hommes. L'intérêt porté aux réalités matérielles s'est accru : les études ostéologiques ont déjà permis de distinguer dans un certain nombre de cas le recours à de jeunes adultes - le fameux bœuf de trois ans des inscriptions - dans les grands sanctuaires et à des animaux prêts à mourir de vieillesse dans les petits sanctuaires ruraux [37]. C'est aussi la question des espaces qui est prise en compte, de façon plus

[28] GINOUVÈs 1962, p. 314

[29] BURKERT 2005, p. 21.

[30] MeULi 1946, p. 254-270.

[31] RUDHARDT 1958, chap. V - les sacrifices sanglants, p. 249-300.

[32] VAN STRATEN 1995

[33] GeORgOUdi 2008, p. 142.

[34] Élien, Sur la nature des animaux, X, 50 et Georgoudi 2008, p. $151-152$.

[35] On peut penser aussi à la cavale fauve qui apparut avant la bataille de Leuctres, quand l'oracle demandait le sacrifice d'une vierge rousse, ce qui sauva les chefs thébains de l'embarras qu'aurait suscité la nécessité d'un sacrifice humain : Plutarque, Pélopidas, 19-20. Les autres sources (Xénophon, Helléniques, VI 4, 7 ; Diodore, XV, 53 et Pausanias, IX, 13, 5-6) ignorent cet épisode.

[36] Le texte de la conférence n'a pas été publié dans les actes du colloque Current approaches to religion in ancient Greece (HAYSOM, WALLENSTEN 2011). Il est simplement fait mention dans la préface ( $\mathrm{p}$. 7) de la stimulante conférence inaugurale.

[37] Sur l'apport fondamental de ces études, voir dans une autre perspective, rituelle, et non socio-économique ЕКROTH 2009. 
concrète encore que lors du colloque de Lyon de 1988 : ainsi S. Huber s'est intéressée en 2011 dans son mémoire inédit d'habilitation à la réalisation des hécatombes [38]. Un travail a débuté en 2013 [39] sur la question matérielle de la mise à mort d'un grand nombre d'animaux et de la consommation de leurs chairs lors des Pythia à Delphes. Comme les travaux menés à Olympie par A. Mallwitz ont montré de façon convaincante le rôle joué par les prairies au sud-est de I'Altis [40], un examen attentif des espaces autour de l'autel d'Apollon à Delphes devrait se révéler fructueux, même si les Delphiens ne bénéficiaient d'une situation aussi favorable que celle qu'offraient les étendues planes entre I'Altis et le cours de l'Alphée. Hérodote parle des broches faites pour transpercer des morceaux de viande de bœuf (obeloi bouporoi) [41] que la courtisane Rhodôpis avait consacrées à Delphes en dîme de ses revenus professionnels et qu'il avait vraisemblablement vues, puisqu'il écrit qu'« elles sont encore aujourd'hui entassées derrière I'autel consacré par les gens de Chios en face du temple même » [42]. Les indications de l'historien invite à regarder vers l'est, dans un secteur qui devait être de plain-pied avec le temple et l'autel lorsque cette offrande, à la fois remarquable et utile [43], fut consacrée ; quand Hérodote la vit, I'aménagement de la terrasse du temple intégrant l'autel
I'avait d'une certaine façon séparée de son contexte originel (le sacrifice), comme c'était le cas de la consommation de viande dans le cadre de laquelle elle était utilisée, activité qui avait lieu sur la terrasse à l'est qui commençait à être occupée par des monuments qui empiétaient sur l'espace offert aux commensaux.

S'il fallait chercher une moralité à cette fable du sacrifice grec, ce pourrait être la suivante : «Ne redis pas ce que tout le monde proclame, mais vois par toi-même, en te confrontant aux documents eux-mêmes » ou « quand théorie et document ne s'accordent pas, garde-toi de l'automne chinois » [44].

[38] HUBER 2011.

[39] Voir supra, n. 16.

[40] MALLWITZ 1999, p. 10-21.

[41] Il ne saurait en effet être question d'embrocher un bœuf entier et de tourner la broche.

[42] Hérodote, II, 135.

[43] La courtisane offrit des objets utiles au culte comme Crésus.

[44] Dans son roman L'Automne à Pékin, B. Vian présente un archéologue qui brise les tessons pour les faire entrer dans ses boîtes.

Figure 1 : L'autel d'Apollon offert à Delphes par la cité de Chios - reconstitution D. Laroche

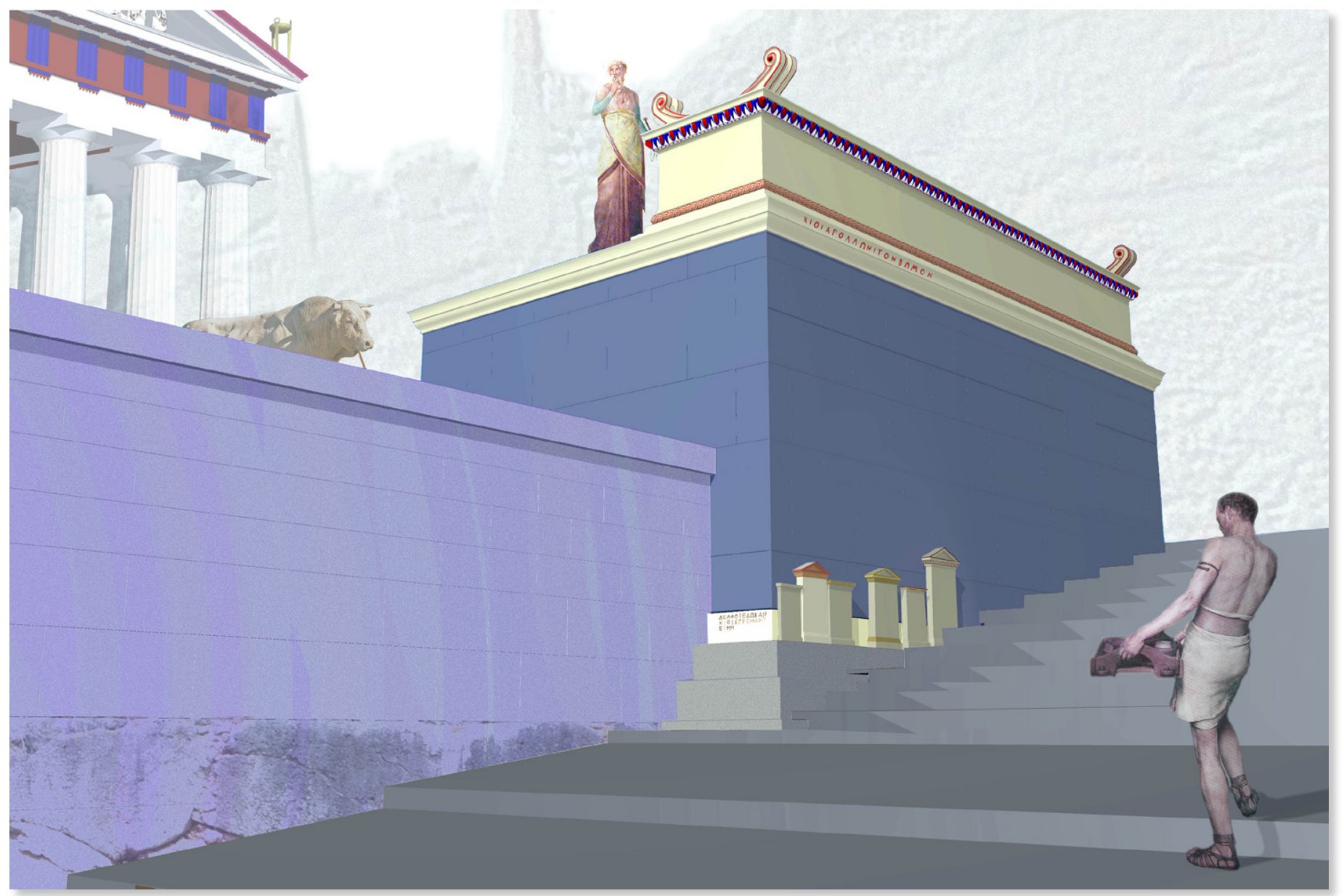


Bruit Zaidman, L., 2001, Le commerce des dieux. Eusebeia. Essai sur la piété en Grèce ancienne, Paris.

Bruit Zaidman, L., Schmitt Pantel, P., 1989 (éd.), La religion grecque, Paris.

BrulÉ, P., 2007A, « La cité et ses composantes : remarques sur les sacrifices et la procession des Panathénées 》, dans Brulé 2007b, p. 231-254 (version remaniée de l'article de Kernos 9 [1996], p. 37-63).

BRuLÉ, P., 2007B, La Grèce d'à côté. Réel et imaginaire en miroir en Grèce antique, Rennes.

BrulÉ, P., 2009 (éd.), La norme en matière religieuse en Grèce ancienne (Kernos Supplément 21), Liège.

Brulé P., Touzé, R., 2008, « Le hiereion : phusis et psuchè d'un medium », dans MehL, Brulé 2008, p. 111-138.

BURKert W., 1972, Homo Necans. Interpretationen altgriechischer Opferriten und Mythen, Bâle-New York.

Burkert, W., 2005, Homo necans : rites sacrificiels et mythes de la Grèce ancienne (tr. fr de Burkert 1972), Paris.

Chantraine, P., 2009, Dictionnaire étymologique de langue grecque, Paris, nouvelle édition.

Сомsтоск, M., et al. s.d., The Trojan War in Greek Art. A Picture Book, Museum of Fine Arts Boston (Mass.).

Detienne, M., Vernant, J.-P., 1972, La cuisine du sacrifice, Paris.

DuRAND, J.-L., 1986, Sacrifice et labour en Grèce ancienne. Essai d'anthropologie religieuse, Paris-Rome.

Durand, J.-L., 1991, « Images pour un autel » dans Étienne, Le Dinahet 1991, p. 45-55.

EкRотH, G., 2009, « Thighs or tails ? The osteological evidence as a source for Greek ritual norms », dans BRULÉ 2009, p. 125-151.

Étienne, R., Le Dinahet, M-Th., 1991 (éd.), L'espace sacrificiel dans les civilisations méditerranéennes de l'Antiquité, Actes du colloque tenu à la Maison de l'Orient, Lyon, 4-7 juin 1988, Lyon.

Georgoudi, St., 2005, « L'occultation de la violence» dans le sacrifice grec : données anciennes, discours modernes », dans Georgoudi, Koch-Piettre, Schmidt 2005, p. 115-147.

Georgoudi, St., 2008, « Le consentement de la victime sacrificielle : une question ouverte », dans MehL, BRULÉ 2008, p. 139-153.

Georgoudi, St., Koch-Piettre, R., Schmidt, F., 2005 (éd.), La cuisine et l'autel. Les sacrifices en question dans les sociétés de la Méditerranée ancienne, Turnhout, 2005.

Ginouvès, R., 1962, Balaneutiké, Recherches sur le bain dans l'Antiquité grecque (BEFAR 200), Paris.

Haysom, Wallensten 2011 : Current approaches to religion in ancient Greece, Papers presented at a symposium at the Swedish Institute at Athens, 17-19 April 2008, edited by M. Haysom and J. Wallensten, Stockholm, 2011.

Huber, S., 2011, Les hécatombes dans les pratiques sacrificielles antiques. Sacrifices publics et marché de la viande dans les cités grecques, mémoire inédit d'habilitation, Lille.

Jost, M., 1992, Aspects de la vie religieuse en Grèce, du début du ve siècle à la fin du III siècle av. J.-C., Paris.

Mallwitz, A., 1999, « Die Ausgrabungen im Südosten (1978 bis 1980) », XI. Bericht über die Ausgrabungen in Olympia, BerlinNew York.

Mehl, V., Brulé P., 2008 (éd.), Le sacrifice antique. Vestiges, procédures et stratégies, Rennes.

Meuli , K., 1946, « Griechische Opferbräuche », dans Phyllobolia. Festschrift Peter von der Mühl, Bâle.

Pirenne-Delforge, Prescendi 2011, «Introduction : «Nourrir les dieux?» », dans V. Pirenne-Delforge et Fr. Prescendi (éds), Nourrir les dieux ? Sacrifice et représentation du divin, Liège, p. 7-14.

RUDHARDT, J., 1958, Notions fondamentales de la pensée religieuse et actes constitutifs du culte en Grèce ancienne, Genève.

Rudhardt, Reverdin 1981 : Le sacrifice dans l'Antiquité, entretiens préparés par J. Rudhardt et O. Reverdin, VandœuvresGenève.

ThomASSEN, E., 2004, « Sacrifice : Ritual murder or dinner party », dans WREDE 2004, p. 275-285.

Van Straten, F., 1995, Hiera Kala. Images of Animal Sacrifice in Archaic and Classical Greece (Roman and Greek Religious World, 127), Leyde-New York-Cologne.

Vernant, J.-P., 1972, «À la table des hommes. Mythe et fondation du sacrifice chez Hésiode », dans Detienne, Vernant 1972, p. $37-132$.

VERnANT, J.-P., 1981, « Théorie générale du sacrifice et de la mise à mort dans la thusia grecque », dans RUDHARD, REVERDIN 1981, p. 1-21 et discussion p. 22-39.

WREDE, M., 2004 (éd.), Celebrations. Selected papers and discussions from the Tenth Anniversary Symposion of the Norwegian Institute at Athens, 12-16 May 1999, Bergen. 